\title{
SPACES DEFINED BY SEQUENCES
}

\author{
ANDREW J. BERNER
}

\begin{abstract}
The biquotient, countably biquotient, hereditarily quotient, and quotient images of $q$-spaces are classified. Also, the quotient images of paracompact $M$-spaces, and the quotient images of $M$-spaces are classified without any separation axioms. New definitions are given for certain familiar classes of spaces to give the definitions more uniformity.
\end{abstract}

1. Introduction. In [1], Michael discussed various generalizations of first countable spaces and gave an extensive collection of theorems providing some answers to the question: "What kind of spaces are the images of various familiar classes of spaces under certain types of quotient maps?" Olson [6] continued this investigation and extended it to some similarly defined classes of spaces which generalize the $q$-spaces introduced in [2]. Most of the spaces involved are defined by conditions requiring the existence of certain types of nested, or decreasing, sequences of sets.

In this paper, we give all the definitions in terms of sequences of sets. Many of the definitions appear as equivalents in [1]. Some of these definitions are, perhaps, simpler than the original, equivalent, definitions, and others are more complicated. The advantage of the scheme lies in the uniformity of the definitions, giving rise to a general construction of pre-images that proves many of the classification theorems of [1]. The same construction also provides a way of classifying the spaces introduced in [6], as well as the quotient images of paracompact $M$-spaces, $M$-spaces and $q$-spaces, without any separation axioms.

In $\S 2$, we define the spaces and the maps in question, and begin to show the relationship between the new spaces introduced and the more familiar class of $k$-spaces. $\$ 3$ gives the construction that will be used in the classification theorems. $\$ 4$ answers the question left open by Olson in [6, p. 6]: "Is every relatively bi-quasi- $k$ (relatively countably bi-quasi- $k$, relatively singly bi-quasi- $k$ ) space a biquotient (countably biquotient, hereditarily quotient) image of a $q$-space?" (The answer is “yes".) $\$ 5$ provides classifications of the quotient images of spaces of pointwise countable type, strict $q$-spaces (and, as corollaries, using theorems of [1], paracompact $M$-spaces and $M$-spaces) and $q$-spaces, without assuming any separation axioms. As a corollary (again using theorems of [1]) we get a further relationship between the spaces introduced for these classifications and $k$-spaces. $\S 6$ raises a question con-

Received by the editors February 10, 1975.

AMS (MOS) subject classifications (1970). Primary 54C10, 54D50, 54D99; Secondary 54D55, 54D 10 .

Key words and phrases. Quotient map, biquotient map, hereditarily quotient map, $k$-space, $q$-space, relatively bi-quasi- $k$-space, $M$-space, pointwise countable type, strict $q$-space.

(C) American Mathematical Society 1976 
cerning regular spaces suggested by these classifications and by a question from [6].

All sets referred to are nonempty sets, and all maps are continuous surjections. All sequences of sets $\left(S_{i}\right)$ are assumed to be decreasing sequences of nonempty sets. No separation axioms are assumed unless specifically stated (in particular, compact and countably compact sets need not be Hausdorff), but regular spaces and normal spaces are assumed to be Hausdorff.

\section{Definitions.}

Definition 2.1. If $S$ is a subset of a topological space $X$, then a decreasing sequence $\left(S_{0} \supset S_{1} \supset S_{2} \supset \cdots\right)$ ) of subsets of $X$ is an $S$-sequence if and only if $s_{i} \in S_{i}$ for all $i$ implies that the sequence $\left(s_{0}, s_{1}, \cdots\right)$ has an accumulation point $^{1}$ in $S$.

(EASy) Examples. (1) A relative $q$-sequence (see [6, Definition 2.3, p. 4]) is an $X$-sequence.

(2) Suppose a sequence of points $\left(s_{i}\right)$ converges to a point $s$ in $X$. Then if $S_{i}=\left\{s_{j}: j \geqslant i\right\}$, the sequence $\left(S_{i}\right)$ is an $\{s\}$-sequence.

REMARK 2.2.(a) If $\left(S_{i}\right)$, a decreasing sequence of subsets of $X$, is an $S$-sequence, and $\left(T_{i}\right)$ is a decreasing sequence with $T_{i} \subset S_{i}$ for each $i$, then $\left(T_{i}\right)$ is also an $S$-sequence.

(b) If $\left(S_{i}\right)$ is an $S$-sequence, and $T \supset S$, then $\left(S_{i}\right)$ is also a $T$-sequence.

The next proposition shows the relationship of $S$-sequences to $k$-sequences and $q$-sequences (see [6, Definition 2.3, p. 4]).

Proposition 2.3. (a) If $\left(S_{i}\right)$ is an $S$-sequence, then $\left(S_{i}\right)$ converges $^{2}$ to $S$.

(b) If $S$ is countably compact, and $\left(S_{i}\right)$ converges to $S$, then $\left(S_{i}\right)$ is an S-sequence.

Proof. (a) Suppose $\left(S_{i}\right)$ is an $S$-sequence, and $O$ is an open set containing $S$. If, for each $i$, there is a point $s_{i} \in S_{i}-O$, then the sequence $\left(s_{i}\right)$ would not have an accumulation point in $S$. So for some $i, S_{i} \subset O$.

(b) This is effectively the content of [4, Lemma 3.1, p. 335].

Corollary 2.4. (a) $\left(S_{i}\right)$ is a $\{p\}$-sequence and $S_{i} \cup\{p\}$ is open for each $i$ if and only if $\left(S_{i} \cup\{p\}\right)$ is a decreasing basis for the open sets at $p$.

(b) $\left(S_{i}\right)$ is an $S$-sequence and $S$ is countably compact if and only if $\left(S_{i} \cup S\right)$ is a q-sequence with $S$ being the countably compact subset of the definition.

(c) $\left(S_{i}\right)$ is an $S$-sequence and $S$ is compact if and only if $\left(S_{i} \cup S\right)$ is a $k$-sequence with $S$ being the compact set of the definition.

In the following group of definitions, the starred $\left(^{*}\right)$ classes of spaces are defined in [6, pp. 6-7]. If the present definition is not the same as in [6], the equivalence is either proved in [1] or is easy to show.

\footnotetext{
${ }^{1}$ A point is an accumulation point of $\left(s_{i}\right)$ iff every neighborhood of the point contains $s_{i}$ for infinitely many $i$.

${ }^{2} \mathrm{~A}$ decreasing sequence of sets $\left(A_{i}\right)$ converges to a set $A$ iff every open set containing $A$ also contains some $A_{i}$ (and thus all but finitely many $A_{i}$ 's).
} 
Definition 2.5. A space $X$ is first countable* (of pointwise countable type*, strict $q^{*}, q$-space s $^{*}$ if each point $p \in X$ has an $S$-sequence $\left(S_{i}\right)$ of open neighborhoods with $S=\{p\}$ ( $S$ compact and $S \subset S_{i}$ for each $i, S$ countably compact and $S \subset S_{i}$ for each $i, S=X$ ).

Definition 2.6. A space $X$ is bisequential* (bi-k*, bi-quasi-k*, relatively bi-quasi- $\left.k^{*}\right)$ if whenever a filter base $\mathscr{F}$ accumulates at a point $p$ in $X$ (i.e. whenever $p \in \bar{F}$ for each $F$ in $\mathscr{F})$, there is an $S$-sequence $\left(S_{i}\right)$ which meshes with $\mathscr{F}$ (i.e. $S_{i} \cap F \neq \varnothing$ for each $i$ and each $F$ in $\left.\mathscr{F}\right)$ with $S=\{p\}(S$ compact, $S$ countably compact, $S=X$ ).

REMARK 2.7. We can, and do, require, without loss of generality, that in the above definitions $p \in \operatorname{Cl}\left(S_{i} \cap F\right)$ for each $i$ and each $F \in \mathcal{F}$, since $\mathscr{F} \cup$ \{neighborhoods of $p$ \} is also a filter base accumulating at $p$.

Definition 2.8. A space $X$ is countably bisequential* (countably bi- $k^{*}$, countably bi-quasi- $k^{*}$, relatively countably bi-quasi- $\left.k^{*}\right)$ if whenever $\left(F_{i}\right)$ is a decreasing sequence accumulating at $p$ in $X$, there is an $S$-sequence $\left(S_{i}\right)$ accumulating at $p$ with $S_{i} \subset F_{i}$ for each $i$ and $S=\{p\}$ ( $S$ compact, $S$ countably compact, $S=X$ ).

DeFinition 2.9. A space $X$ is Fréchet* (singly bi-k*, singly bi-quasi- $k^{*}$, relatively singly bi-quasi-k*) if whenever $p \in \bar{F}$, there is an $S$-sequence $\left(S_{i}\right)$ accumulating ai $p$ with $S_{i} \subset F$ for each $i$ and $S=\{p\}$ ( $S$ compact, $S$ countably compact, $S=X$ ).

Definition 2.10. A space $X$ is sequential* (sequentially- $k$, sequentially quasi- $k$, sequentially $-q)^{3}$ if whenever $F$ is not closed in $X$, there is a point $p \in \bar{F}-F$ and an $S$-sequence $\left(S_{i}\right)$ accumulating at $p$ with $S_{i} \subset F$ for each $i$ and $S=\{p\}$ ( $S$ compact, $S$ countably compact, $S=X)$.

Most of the implications among these classes of spaces have been reported in [1] and [6]. Among the new classes defined, it is obvious that $X$ is sequential $\Rightarrow X$ is sequentially- $k \Rightarrow X$ is sequentially quasi- $k \Rightarrow X$ is sequentially- $q$. (Of course, the similar implications among the spaces in Definitions 2.5, 2.6, 2.8 and 2.9 are just as obvious.) Also, every singly bi- $k$ space is sequentially- $k$, every singly bi-quasi- $k$ space is sequentially quasi- $k$, and every relatively singly bi-quasi- $k$ space is sequentially- $q$.

Proposition 2.11. Every $k$-space (quasi-k-space) is sequentially-k (sequentially quasi-k). (See [1] or [6] for definitions!)

Proof. If $X$ is a $k$-space (quasi- $k$-space) and $F \subset X$ is not closed, then there is a compact (countably compact) set $S$ and a point $p \in S$ which is also in $\mathrm{Cl}(F \cap S)-F$. But if $S_{i}=F \cap S$ for each $i$, then $p,\left(S_{i}\right)$ and $S$ are as required by the definition of a sequentially- $k$ (sequentially quasi- $k$ ) space.

We now give definitions of four types of quotient maps in the same spirit as the definitions of the spaces.

Definition 2.12 [3, Proposition 2.2, p. 290]. A map $f: X \rightarrow Y$ is biquotient if whenever a filter base $\mathscr{F}$ accumulates at $y$ in $Y$, then $f^{-1}(\mathscr{F})$ accumulates at some $x \in f^{-1}(y)$.

Definition 2.13 [7, Proposition 3.2, p. 149]. A map $f: X \rightarrow Y$ is countably biquotient if whenever $\left(A_{i}\right)$ is a decreasing sequence accumulating at $y$ in $Y$, then $\left(f^{-1}\left(A_{i}\right)\right)$ accumulates at some $x \in f^{-1}(y)$.

\footnotetext{
${ }^{3}$ The terminology was suggested by E. Michael.
} 
Definition 2.14 [1, Lemma 5.2, p. 115]. A map $f: X \rightarrow Y$ is hereditarily quotient if whenever $y \in \bar{A}$ in $Y$ then $x \in \mathrm{Cl}\left(f^{-1}(A)\right)$ for some $x \in f^{-1}(y)$.

Definition 2.15. A map $f: X \rightarrow Y$ is quotient if whenever $A$ is not closed in $Y$, then for some $y \in \bar{A}-A$, and for some $x \in f^{-1}(y), x \in \mathrm{Cl}\left(f^{-1}(A)\right)$.

REMARK 2.16. Definition 2.15 is obviously equivalent to the usual definition of "quotient map" and points out the relationship between quotient maps and the spaces defined in Definition 2.10.

3. The main construction. The following construction is the heart of the proofs of the classification theorems in $\S \S 4$ and 5 .

Definition 3.1. If $\left(S_{i}\right)$ is a decreasing sequence of subsets of a topological space $X$, we define a new topology on $X$ as follows. A basic open set around a point $p$ in $X$ is any set of the form $U \cap\left(\{p\} \cup S_{i}\right)$ where $p \in U$ and $U$ is open in the original topology on $X$. $X$, with this new topology, is called $X\left(S_{i}\right)$.

REMARK 3.2. We will need these facts about $X\left(S_{i}\right)$.

(a) The identity map: $X\left(S_{i}\right) \rightarrow X$ is continuous.

(b) If $p \in X$ and $i \in \omega$, then $\{p\} \cup S_{i}$ is open in $X\left(S_{i}\right)$.

(c) If $F \subset X$, then

$$
\begin{array}{r}
\left.\bigcap \text { closure in } X \text { of } S_{i} \cap F: i \in \omega\right\} \\
=\bigcap\left\{\text { closure in } X\left(S_{i}\right) \text { of } S_{i} \cap F: i \in \omega\right\} .
\end{array}
$$

(d) If $s_{i} \in S_{i}$ for each $i$, and $s$ is an accumulation point of $\left(s_{i}\right)$ in the original topology on $X$, then $s$ is still an accumulation point of $\left(s_{i}\right)$ in $X\left(S_{i}\right)$.

(e) If the original topology on $X$ is Hausdorff, then so is $X\left(S_{i}\right)$.

(f) The relative topology on $\cap\left\{S_{i}: i \in \omega\right\}$ is the same in both $X\left(S_{i}\right)$ and in $X$.

REMARK 3.3. In contrast to (e) above, $X\left(S_{i}\right)$ need not be regular even if $X$ is. This will give rise to some questions in $\S 6$.

Proposition 3.4. If $\left(S_{i}\right)$ is an $S$-sequence in $X$, then $\left(S_{i}\right)$ is still an $S$-sequence in $X\left(S_{i}\right)$.

Proof. This follows immediately from Remark 3.2(d).

Proposition 3.5. If $\left(S_{i}\right)$ is a relative $q$-sequence ( $q$-sequence, $k$-sequence) in $X$, then it still is in $X\left(S_{i}\right)$.

Proof. If $\left(S_{i}\right)$ is a relative $q$-sequence in $X$ (i.e. an $X$-sequence) then Proposition 3:4 immediately implies that $\left(S_{i}\right)$ is a relative $q$-sequence in $X\left(S_{i}\right)$. If $\left(S_{i}\right)$ is a $q$-sequence ( $k$-sequence) in $X$, then it follows from Proposition 3.4, Remark 3.2(f), and Corollary 2.4(b) (Corollary 2.4(c)) that $\left(S_{i}\right)$ is a $q$-sequence (k-sequence) in $X\left(S_{i}\right)$.

\section{Images of $q$-spaces.}

THEOREM 4.1. A space $Y$ is a relatively bi-quasi- $k$ space if and only if there is a $q$-space $X$ and a biquotient map $f: X \rightarrow Y$.

Proof. As remarked in [6, p. 6], every biquotient image of a $q$-space is routinely seen to be relatively bi-quasi- $k$. Suppose, then, that $Y$ is relatively bi-quasi- $k$. For each $y \in Y$ and filter base $\widetilde{F}$ accumulating at $y$, choose a 
$Y$-sequence $\left(Q_{0}(y, \mathscr{F}) \supset Q_{1}(y, \mathscr{F}) \supset \cdots\right)$ that meshes with $\mathscr{F}$, with $y \in$ $\mathrm{Cl}\left(Q_{i}(y, \mathscr{F}) \cap F\right)$ for each $i$ and each $F \in \mathscr{F}$ (see Remark 2.7). Consider the space $Y(y, \mathscr{F})=Y\left(Q_{i}(y, \mathscr{F})\right)$ as defined by Definition 3.1. Let $X$ be the disjoint union of the spaces $Y(y, \mathscr{F})$ for each such pair $(y, \mathscr{F})$, and let $f$ : $X \rightarrow Y$ be defined by: $f \mid Y(y, \mathscr{F})$ is the identity. By Remark 3.2(a), $f$ is continuous.

Claim 1. $X$ is a $q$-space.

Proof. This follows from Remark 3.2(b), Proposition 3.4, and Remark 2.2(b).

Claim 2. The map $f$ is biquotient.

Proof. Suppose $\mathscr{F}$ accumulates at a point $y$ in $Y$. Since for any $F \in \mathscr{F}$, the sequence $\left(Q_{i}(y, \mathcal{F}) \cap F\right)$ accumulates at $y \in Y$, then, by Remark 3.2(c), the sequence $\left(f^{-1}\left(Q_{i}(y, \mathscr{F})\right) \cap Y(y, \mathscr{F}) \cap f^{-1}(F)\right)$ accumulates at $y \in$ $Y(y, \mathscr{F})$ (formally, this point is the unique element of $f^{-1}(y) \cap Y(y, \mathscr{F})$ ). So $f^{-1}(\mathscr{F})$ accumulates at $y \in Y(y, \mathscr{F})$ proving $f$ is biquotient.

Claims 1 and 2 prove that $X$ and $f$ are the space and the map required by the theorem.

THEOREM 4.2. A space $Y$ is a relatively countably bi-quasi- $k$ space if and only if there is a q-space $X$ and a countably biquotient map $f: X \rightarrow Y$.

Proof. One direction is again routine. (See Theorem 5.1 for an example of a verification of this sort!) In fact, all countably biquotient images of relatively countably bi-quasi- $k$ spaces are relatively countably bi-quasi- $k$. Now suppose $Y$ is a relatively countably bi-quasi- $k$ space. For each $y$ in $Y$ and each decreasing sequence $\left(F_{i}\right)$ accumulating at $y$, choose a $Y$-sequence $\left(S_{i}\right)$ with $S_{i} \subset F_{i}$ for each $i$, and $\left(S_{i}\right)$ accumulating at $y$, and proceed as in the proof of Theorem 4.1.

THEOREM 4.3. A space $Y$ is a relatively singly bi-quasi-k space if and only if there is a q-space $X$ and a hereditarily quotient map $f: X \rightarrow Y$.

Proof. Similar to the proofs of Theorems 4.1 and 4.2.

5. Quotient images of spaces. In [1, p. 120], Michael got partial characterizations of quotient images of paracompact $M$-spaces and $M$-spaces by looking at $k$ and quasi- $k$ spaces. However, some separation axioms were required. We now prove that the spaces defined in Definition 2.10 are precisely the right classes of spaces to look at.

THEOREM 5.1. A space $Y$ is sequentially- $k$ if and only if there is a space $X$ of pointwise countable type and a quotient map $f: X \rightarrow Y$.

Proof. Suppose $X$ is of pointwise countable type, $f: X \rightarrow Y$ is quotient, and $A \subset Y$ is not closed. Then there is a $y \in \bar{A}-A$ and an $x \in f^{-1}(y)$ with $x \in \mathrm{Cl}\left(f^{-1}(A)\right)$. Let $\left(S_{i}\right)$ be an $S$-sequence of open neighborhoods of $x$ with $\cap\left\{S_{i}: i \in \omega\right\} \supset S$, a compact set in $X$. Consider $\left(f\left(S_{i}\right)\right)$. Clearly this is an $f(S)$-sequence and $f(S)$ is compact in $Y$. So by Remark 2.2(a), $\left(f\left(S_{i}\right) \cap A\right)$ is also an $f(S)$-sequence. If $O$ is an open set containing $y$, then $f^{-1}(O) \cap S_{i}$ is a neighborhood of $x$ for each $i$, and thus $f^{-1}(O) \cap S_{i} \cap f^{-1}(A) \neq \varnothing$. Thus $O \cap f\left(S_{i}\right) \cap A \neq \varnothing$, so the sequence $\left(f\left(S_{i}\right) \cap A\right)$ accumulates at $y$. Thus $Y$ is sequentially- $k$. 
Assume now that $y$ is sequentially- $k$. For each set $A$ that is not closed, pick a $y \in \bar{A}-A$ and an $S(A)$-sequence $\left(S_{i}(A)\right)$ accumulating at $y$ with each $S_{i}(A) \subset A$ and $S(A)$ compact. Define $Y(A)=Y\left(S_{i}(A) \cup S(A)\right)$ as defined by Definition 3.1 and let $X$ be the disjoint union of the spaces $Y(A)$ such that $A$ is not closed in $Y$. Let $f: X \rightarrow Y$ be defined by: $f \mid Y(A)$ is the identity. By Remark 3.2(a), $f$ is continuous.

Claim 1. $X$ is a space of pointwise countable type.

Proof. If $p \in X$, then $p \in Y(A)$ for some $A$. By Remark 3.2(b) $\{p\} \cup$ $S_{i}(A) \cup S(A) \subset Y(A)$ is open in $Y(A)$ (thus in $X$ ) for each $i$, and by Corollary 2.4(c) and Proposition 3.5, $\left(\{p\} \cup S_{i}(A) \cup S(A)\right)$ is a $k$-sequence in $Y(A)$ (thus in $X$ ); i.e. $\left(\{p\} \cup S_{i}(A) \cup S(A)\right)$ is an $S(A) \cup\{p\}$-sequence and $S(A) \cup\{p\}$ is compact in $X$ as required.

Claim 2. The map $f$ is quotient.

Proof. Suppose $A$ is not closed in $Y$. Then we chose a point $y \in \bar{A}-A$ in defining $Y(A)$. Consider $y \in Y(A)$ (i.e. $\{y\}=f^{-1}(y) \cap Y(A)$ ). Suppose $U \cap\left(\{y\} \cup S_{i}(A) \cup S(A)\right)$ is a basic open set in $X$ around $y$. Since the sequence $\left(S_{i}(A)\right)$ accumulates at $y \in Y$, there is an $x \in U \cap S_{i}(A)$. Look at $x \in Y(A)$. Since $f(x)=x, S_{i}(A) \subset A$, and $x \in U \cap\left(\{y\} \cup S_{i}(A) \cup S(A)\right)$, it follows that

$$
x \in f^{-1}(A) \cap\left(U \cap\left(\{y\} \cup S_{i}(A) \cup S(A)\right)\right) .
$$

Thus $y \in Y(A)$ is in $\mathrm{Cl}\left(f^{-1}(A)\right)$, so $f$ is quotient.

Claims 1 and 2 show that $X$ and $f$ are the space and the map required by the theorem.

Corollary 5.2. A space is sequentially-k if and only if it is the quotient image of a paracompact $M$-space.

Proof. By [1, Theorem 3.E.3, p. 107], every space of pointwise countable type is a biquotient image, thus a quotient image, of a paracompact $M$-space, and the composition of quotient maps is quotient. Conversely, by [1, Proposition 2.E.3, p. 103] (or as is easily shown directly), every paracompact $M$-space is a space of pointwise countable type, and thus all its quotients are sequentially- $k$.

COROllary 5.3. Every Hausdorff sequentially-k space is a $k$-space.

Proof. If $Y$ is a Hausdorff sequentially- $k$ space, then the space $X$ constructed in the proof of Theorem 5.1 is also Hausdorff by Remark 3.2(e). Then, by [8, Theorem 1, p. 138], $X$ is the open image of a Hausdorff paracompact $M$-space, so $Y$ is the quotient image of such a space. So by [1, Theorem 6.E.3b, p. 120] $Y$ is a $k$-space.

THEOREM 5.4. A space $Y$ is sequentially quasi- $k$ if and only if there is a strict $q$-space $X$ and a quotient map $f: X \rightarrow Y$.

Proof. The proof is essentially the same as for Theorem 5.1.

Corollary 5.5. A space is sequentially quasi-k if and only if it is the quotient image of an $M$-space.

Proof. The proof is similar to Corollary 5.2, using [1, Theorem 3.F.3, p. 
109] instead of [1, Theorem 3.E.3], and [1, Proposition 2.F.3, p. 104] instead of [1, Proposition 2.E.3].

THEOREM 5.6. $A$ space $Y$ is sequentially- $q$ if and only if there is a q-space $X$ and a quotient map $f: X \rightarrow Y$.

Proof. Modify the proof of Theorem 5.1 as follows. For each $A$ not closed in $Y$, choose a point $y \in \bar{A}-A$ and a $Y$-sequence $\left(S_{i}(A)\right)$ accumulating at $y$ with $S_{i} \subset A$ for each $i$. Now define $Y(A)$ to be, simply, $Y\left(S_{i}(A)\right)$ and proceed as in the proof of Theorem 5.1.

THEOREM 5.7. Every regular sequentially quasi-k space is a quasi-k space.

Proof. (This theorem appeared as a question in the original typescript of this paper. This answer was provided by R. C. Olson.) Suppose $A \subset Y$ is not closed. Then there is a $p \in \bar{A}-A$ and an $S$-sequence $\left(S_{i}\right)$ accumulating at $p$ with $S_{i} \subset A$ for each $i$, and $S$ countably compact. We can assume $p \in S$. If $A \cap S$ is closed in $S$, then there are open sets $U$ and $V$ with $p \in U$, $\mathrm{Cl}(A \cap S) \subset V$ and $U \cap V=\varnothing$. Choose $s_{i} \in S_{i} \cap U$. Then $S \cup\left\{s_{i}\right.$ : $i \in \omega\}$ is countably compact, and $A \cap\left(S \cup\left\{s_{i}: i \in \omega\right\}\right)$ is not closed in $S \cup\left\{s_{i}: i \in \omega\right\}$.

COROLlaRY 5.8. Every regular sequentially quasi-k space is the quotient image of a regular strict $q$-space.

Proof. This follows from Theorem 5.7 and the fact that a quasi- $k$ space is the quotient image of the disjoint union of its countably compact subsets.

6. Questions about regular sequentially- $q$ spaces. Corollary 5.8 raises the following question:

(1) Is every regular sequentially- $q$ space the quotient image of a regular $q$-space?

Also, we can add to [6, Problem 2.9, p. 8]:

(2) Is every regular sequentially- $q$ space a sequentially quasi- $k$ space?

Question 2 is interesting, in that, as in [6], the answer is "yes" if regular is replaced by normal, but "no" if regular is replaced by Hausdorff. In light of Corollary 5.8, and the fact that regular $q$-spaces are strict $q$-spaces, Questions 1 and 2 are equivalent. Since this paper was originally written, an example has been found showing that the continuum hypothesis implies the answer to these questions is "no."

\section{REFERENCES}

1. E. A. Michael, A quintuple quotient quest, General Topology and Appl. 2(1972), 91-138. MR46 \#8156.

2. __ A note on closed maps and compact sets, Israel J. Math. 2(1964), 173-176. MR31 \# 1659.

3. , Bi-quotient maps and Cartesian products of quotient maps, Ann. Inst. Fourier (Grenoble) 18(1968), fasc. 2, 287-302(1969). MR39 \#6277.

4. _ On representing spaces as images of metrizable and related spaces, General Topology and Appl. 1(1971), 329-343. MR45 \#2681. 
5. J. Nagata, Quotient and bi-quotient spaces of M-spaces, Proc. Japan Acad. 45(1969), 25-29. MR39 \#6278.

6. R. C. Olson, Bi-quotient maps, countably bi-sequential spaces and related topics, General Topology and Appl. 4(1974), 1-28.

7. F. Siwiec, Sequence-covering and countably bi-quotient mappings, General Topology and Appl. 1(1971), no. 2, 143-154. MR44 \#5933.

8. H. H. Wicke, On the Hausdorff open continuous images of Hausdorff paracompact p-spaces, Proc. Amer. Math. Soc. 22(1969), 136-140. MR39 \# 4801.

Department of Mathematics, University of Wisconsin, Madison, Wisconsin 53706 